\title{
ZS Research Square

\section{Knowledge, attitude and practice of clinicians about antimicrobial stewardship and resistance among hospitals of Pakistan: A multicenter cross-sectional study}

Sohaib Ashraf ( $\square$ sashraf1@mgh.harvard.edu )

Massachusetts General Hospital https://orcid.org/0000-0003-3127-3557

Shoaib Ashraf

Riphah International University

Moneeb Ashraf

King Edward Medical College: King Edward Medical University

Muhammad Ahmad Imran

Shaikh Zayed Medical Complex

\section{Zawar Ahmad}

Shaikh Zayed Medical Complex

Hadiqa tul Hafsa

Shaikh Zayed Medical Complex

Abeer Bin Awais

Shaikh Zayed Medical Complex

Larab Kalsoom

Services Institute of Medical Sciences

\section{Rutaba Akmal}

Sahara Medical College

Iqra Farooq

Children's Hospital

Sidra Ashraf

University of Veterinary and Animal Sciences

\section{Zaighum Habib}

THQ Ferozwala

Muhammad Ghufran

SACHS Chile

Syed Sami Hussain Sherazi

University of Veterinary and Animal Sciences

Muhammad Kiwan Akram

University of Veterinary and Animal Sciences 


\section{Sundas Rafique}

Mayo Hospital

Khawar Nawaz

sunny downstate

Zartasha Safdar

University of Veterinary and Animal Sciences

Muhammad Ashraf

University of Veterinary and Animal Sciences

Qazi Abdul Saboor

Shaikh Zayed Medical Complex

Ayesha Humayun

Shaikh Zayed Medical Complex

Mateen Izhar

Shaikh Zayed Medical Complex

ONE Health Consortium

Shaikh Zayed Medical Complex

Research

Keywords: antimicrobials, resistance, antimicrobial use, antibiotic misuse, antibiotic resistance

Posted Date: May 10th, 2021

DOl: https://doi.org/10.21203/rs.3.rs-467849/v1

License: (c) (1) This work is licensed under a Creative Commons Attribution 4.0 International License. Read Full License 


\section{Abstract}

Considering that antimicrobial resistance (AMR) is a global challenge, there is a dire need to gauge the knowledge, attitude, and practice (KAP) of clinicians in endemic countries. The aim of the current multicenter, cross-sectional study was to highlight the knowledge, aptitude and practice gaps in antimicrobial (AM) stewardship and AMR among practicing doctors working in public tertiary care teaching hospitals of Lahore, Pakistan. A KAP survey, based on a self-administered questionnaire containing 45 questions, was conducted among 336 clinicians practicing in 6 randomly selected hospitals of Lahore, Pakistan. Overall, $92 \%$ of the clinicians considered AMR as a worldwide problem but only $66 \%$ disagreed that cold and flu symptoms require antibiotics. Moreover, around $68 \%$ doctors felt confident about their practice in AM but still $96 \%$ felt need to get more knowledge about AM. Need to establish courses on rational antibiotic use was demanded by $84 \%$ of participants. The main contributing factors considered for AMR by the doctors included excessive AM usage in medical profession (87.1\%) and multiple antibiotics per prescription (76.4\%). Pharmacologically, AM spectrum was accurately chosen by $1.4 \%$ (ampicillin), $0.003 \%$ (erythromycin) and $0 \%$ (levofloxacin). Clinically, a more than $50 \%$ of clinicians used miscellaneous AM for empirical therapy of respiratory tract infection and cholecystitis. The data was analyzed using Statistical Package for Social Sciences (SPSS) version 25. The knowledge of clinicians is relatively poor in AM spectrum and drugs of choice for certain infections. However, they know about their short comings with positive approach towards improvement.

\section{Introduction}

Although there were significant advances in antimicrobial therapy in 1980s as the third-generation cephalosporin and new fluoroquinolones proved to be highly effective. The widespread usage of these newly developed drugs soon gave rise to a new problem. A decade down the line, clinicians started to see persistent infections despite the antibiotic regimens. Newer strains of multi-drug resistant staphylococci, enterococci and streptococcus bacteria were identified.(Levy 1998, Okeke, Laxminarayan et al. 2005, Cosgrove 2006, Organization 2012) With emerging antimicrobial resistance (AMR), there is concordant rise in the morbidity and mortality ratios. Accompanying the high mortality and morbidity was the economic stress this problem had on the health care sector in low-income countries. (Organization 2012) Factors responsible for AMR in the third world countries include over-prescribing, unnecessary prescribing, incomplete treatment course, self-medication and insufficient infection control measures to prevent spread of resistant bacteria both in the community and the hospital.(Okeke 2010, Sosa, Byarugaba et al. 2010) The first step to deal with AMR is to educate the related staff about the over expanding AMR dilemma.(Dellit, Owens et al. 2007, Pulcini, Cua et al. 2007) This knowledge has led USA, France and Scotland to immediately develop a national recommendations program to improve antibiotic stewardship in their countries.(Nathwani 2006, Dellit, Owens et al. 2007, Pulcini, Cua et al. 2007) In certain studies, it is known that the misuse of antibiotics by general population increases the chance of getting infected with the resistant strains of bacteria, yielding higher morbidity and mortality. (Cabana, Rand et al. 1999, Woodford and Livermore 2009, Costelloe, Metcalfe et al. 2010) Meanwhile, the best 
approach to contain AMR is to minimize the antimicrobial usage and it can be achieved by changes in prescribing behavior.(Wester, Durairaj et al. 2002, Giblin, Sinkowitz-Cochran et al. 2004, Srinivasan, Song et al. 2004, Guerra, Pereira et al. 2007, García, Llamocca et al. 2011, Pulcini, Williams et al. 2011) Factors thriving prescribing behavior of medical doctors can be analyzed by evidence focused questionnaire such as knowledge, attitude and practice (KAP) surveys.

In addition, the available studies have all been in Europe, U.S. China, Brazil, and Peru but few studies were done in Pakistan.(Wester, Durairaj et al. 2002, Giblin, Sinkowitz-Cochran et al. 2004, García, Llamocca et al. 2011, Faizullah, Nisar-ur-Rahman et al. 2017, Shahid, Iftikhar et al. 2017, Ahmed, Bhimani et al. 2020) Therefore, we conducted a KAP-survey about antimicrobial usage and antimicrobial resistance among public tertiary care teaching hospitals of Lahore, Pakistan.

\section{Methodology}

It was a cross-sectional study in which a self-administered questionnaire was distributed among physicians at the postgraduate trainee level and faculty in six randomly selected tertiary care teaching hospitals of Lahore, Pakistan. The variables encountered were knowledge of doctors regarding antimicrobial usage and its resistance, their attitude towards antimicrobial usage and resistance, and their practice concerning both. The inclusion criteria included clinicians working in medicine, surgery, and their allied departments. Psychiatrists, radiologists and basic sciences doctors were excluded. The questionnaire was distributed on-site during working hours in hard copy along with the option of filling it digitally.

The questionnaire was developed in consultation with a group of experts on questionnaire design and infectious diseases, and after searching the literature for comparable studies.(Wester, Durairaj et al. 2002, Srinivasan, Song et al. 2004, Guerra, Pereira et al. 2007) Before we finalized the questionnaire, it was reviewed by a panel of clinical experts of Shaikh Zayed Post-Graduate Medical Complex, Lahore, Pakistan. The questionnaire enquired the participants' awareness regarding the recent scope of antimicrobial resistance (15 questions), the clinical attitude contributing to the above-mentioned fact inputs (5 questions), factors influencing confidence regarding usage and prescription of AM and AMR (6 questions), knowledge regarding contributing factors of AMR (7 questions) and basic knowledge about the clinical indications, spectrum, administration and pharmacology of antimicrobials (12 questions). The questions used a 4 or 5-point Likert scale (which included answers ranging from "strongly agree" to "strongly disagree", from "very confident" to "not confident" and from "always" to "never"). The anonymous nature of questionnaire and prior consent form from all clinicians addressed ethical issues. This research was in accordance with principle 3 and 4 of World Medical Association's declaration of Helsinki. The questionnaire was submitted in a pilot test to 34 participants to check comprehension and clarity of the questions.

Randomized selection of tertiary care teaching hospitals of Lahore by using lottery method was done which made us to select Ganga Ram Hospital, Jinnah Hospital, Services Hospital, Mayo Hospital, Sheikh 
Zayed Hospital and General Hospital. Sample size was calculated using www.epitool.ausvet.com with estimated true proportion 0.5 with confidence level of $95 \%$ and desired precision of 0.05 , the population size was calculated to be 2614 doctors. The doctors were contacted after consultation with administrators/ medical superintendents of respective hospitals among the 6 hospitals, and the calculated sample size was 336. It was predefined that a questionnaire was to be considered valid for analysis if $90 \%$ of questions were answered. The data was analyzed using IBS SPSS software version 25 (SPSS Inc., Chicago, IL, USA)

\section{Results}

All participants had at least a Bachelor of Medicine and Bachelor of Surgery (MBBS) degree with mean age of 31 years. Of the total, 336 filled questioners, $84.5 \%$ (284) met the selection criteria. Among those, $34.5 \%$ were males and $25.7 \%$ were faculty members (Table 1). In our study (Fig. 1), 92\% of the clinicians considered AMR as a worldwide problem but only $66 \%$ disagreed that cold and flu symptoms require antibiotics. Though, around $68 \%$ doctors felt confident about their knowledge and practice in the antibiotic prescription but still $96 \%$ felt necessary to get more knowledge about AM. Along with that, $20 \%$ believed AM prescription would not cause damage. Furthermore, 78\% doctors blamed that poor-quality antibiotics made them ineffective, while $35 \%$ believed efficacy would be better with newer costly antibiotics. Furthermore, $19 \%$ thought patients' demand contributed to over-prescription while $17 \%$ considered over-prescription better than under-prescription in infected patients and $37 \%$ agreed antimicrobials should be available over the counter. Moreover, $69 \%$ believed AMR is not a problem in their clinical settings along with $84 \%$ demanded need to establish courses on rational antibiotic use and to carry out large scale antibiotic awareness (39\%)

According to the study (Fig. 2A), antibiotics were being selected confidently by $75.4 \%$ doctors and more than $81 \%$ felt confident while planning duration of AM treatment however $74.3 \%$ felt confident while stopping treatment. More than $75 \%$ of the respondents felt confident while selecting correct dose, duration of administration and using combination therapy. Almost $80 \%$ were also confident that they could choose between intravenous and oral routes correctly.

The factors which were regarded as being important causes of antibiotic resistance included: multiple antibiotics per prescription (76.4\%) and excessive use of AM in daily life (87.1\%). The factors most frequently identified as less important included: prescribing too many broad-spectrum antibiotics (61.7\%), prescribing low dose antibiotics (60.5\%), longer duration of AM usage $(66.2 \%)$ and poor hand hygiene (54.9\%). The factor which was regarded as least important was paying too much attention to advertising (36.3\%) (Fig. 2B).

$65 \%$ of doctors felt mostly confident about prescribing antibiotics while $40 \%$ considered sometimes about AMR while prescribing. Half of the respondents agreed that doctors sometimes got influenced by multinational companies while approximately $1 / 4$ th of the doctor community belonged to the class who believed it occurred more often. $50 \%$ of doctors sometimes, and $30 \%$ most of the times reviewed their 
choice for antibiotic usage with their senior colleagues and $11 \%$ of the times mostly and $71 \%$ sometimes suggested a different antibiotic (Fig. 2C).

Knowledge about the empiric therapy of various common infections was inquired (Fig. 3). For urinary tract infection (uncomplicated), around one half of the doctors prescribed ciprofloxacin, while levofloxacin and TMP-SMZ were written by $11.4 \%$ and $5.6 \%$ respectively as first line treatment. Upper respiratory tract infection was being managed supportively by only $3.9 \%$ of clinicians as per world health organization (WHO) recommendations while one forth were managing via amoxicillin and around $10 \%$ were being treated by levofloxacin and azithromycin cumulatively. Shockingly, more than $70 \%$ were prescribing miscellaneous drugs. In clinical practice, doctors considered ciprofloxacin and metronidazole in around $31 \%$ and $40 \%$ of their prescriptions respectively as first line treatment of gastroenteritis while only $11 \%$ decided to manage it conservatively. First line of choice for community acquired pneumonia by approximately 13 doctors was amoxicillin, levofloxacin and ceftriaxone individually, azithromycin was prescribed by $7 \%$ of them and more than $50 \%$ of prescriptions were contributed by miscellaneous drugs. Most of the clinicians (50\%) prescribed miscellaneous drugs as first line antibiotic while others chose different drugs for treatment of cellulites that include amoxicillin (9.6\%) and ceftriaxone (7.4\%). Also $26.9 \%$ clinicians chose drugs other than these for the said purpose. As far as cholecystitis was concerned, majority disputed on the first line antimicrobial by choosing miscellaneous drugs (57\%), all different agents being prescribed for that.

Knowledge about the antimicrobial spectrum was also evaluated (Fig. 4). 1.4\% of the clinicians chose correct spectrum of Ampicillin.(Rafailidis, loannidou et al. 2007) However, $88.3 \%$ were inclined towards gram positive cocci, $39.2 \%$ considered it to be active against gram positive bacilli, $31.8 \%$ believed it to be active against gram negative cocci, $14.3 \%$ suggested it as a preferred treatment for gram-negative bacilli and $2.6 \%, 10 \%$ and $3.3 \%$ marked anaerobes, spirochetes, and atypical bacteria respectively. Amazingly, the correct antimicrobial spectrum of Metronidazole(Freeman, Klutman et al. 1997) was selected by $61.6 \%$. However, $85 \%$ of doctors do know that it is effective against anaerobes while $13.3 \%$ considered its coverage for gram-positive cocci and other lesser percentages of doctors considered it to be effective against other organisms. Astonishingly, none of the clinicians were able to identify the correct antimicrobial spectrum of Levofloxacin.(Zhanel, Fontaine et al. 2006, Anderson and Perry 2008) On the better side, greater than half of doctors knew its effectiveness against gram positive cocci, more than $1 / 3$ rd considered it effective against gram positive bacilli and gram-negative rods along with that $20 \%$ correctly identified it to be effective against atypical bacteria. However, around $40 \%$ of the doctor community also thought it to be effective against gram-negative cocci. Only one doctor $(0.003 \%)$ came up with the correct antimicrobial spectrum for Erythromycin (Lartey, Nellans et al. 1994, Zhanel, Dueck et al. 2001) but $68.2 \%$ of the doctors considered its coverage against gram positive cocci to be true. Almost $25-35 \%$ of the doctors contemplated its effectiveness against, gram negative bacilli, gram negative cocci, gram positive bacilli and atypical bacteria. Around $5.9 \%$ considered it to be effective against spirochetes but $6.8 \%$ believed it also acted on anaerobes. $0.4 \%$ picked correct spectrum of vancomycin, $77.4 \%$ chosen gram positive cocci while gram negative cocci and gram positive bacilli were chosen by around 33\% clinicians.(Wilhelm and Estes 1999, Marsot, Boulamery et al. 2012) Anaerobes, spirochetes 
and atypical were marked by less than $10 \%$ of doctors. $2.8 \%$ of the doctors opted correct spectrum of Ceftriaxone.(Cleeland and Squires 1984, Lamb, Ormrod et al. 2002) 53.9\% selected gram positive cocci as right option whereas $66.3 \%$ handpicked gram negative cocci. Gram positive bacilli were nominated as the correct option by $29.1 \%$ while $39.1 \%$ saw gram negative bacilli as correct option. Anaerobes $(9.4 \%)$, spirochetes $(11.3 \%)$ and atypical $(10.7 \%)$ were marked.

\section{Discussion}

This survey demonstrated the knowledge, attitude, and practices of doctors regarding antimicrobial resistance in six large public teaching hospitals in Lahore. From the study, it was evident that the knowledge about AMs resistance, their misuse, and the concern regarding their emerging resistance problem worldwide was apt. But despite all this, on ground realities were quite astonishing as most of the physicians were using AMs even for mild upper respiratory tract infections. Moreover, their attitude for prescribing multiple antibiotics per prescription was also questionable. Majority of the doctors' knowledge pertaining to AM spectrum and empirical treatment for certain infections was not up-to the mark. This shows that special attention should be given to this matter and appropriate interventions must be used to improve the knowledge of doctors in this area. It was evident from the study that $91.9 \%$ participants considered AMR to be uprising concern, yet AMR was almost completely ignored in their own practice. Majority agreed on the fact that the main reason for such growing resistance is the daily frequent use of AM. A small ray of sunshine was seen that a number of newer doctors were seen seeking help from their experienced colleagues when prescribing, irrespective of their specialization (department) so that the appropriate antibiotic could be given to avoid resistance. However, $40 \%$ of participants responded that they did not consult their peers. $19.4 \%$ of the participants shed light on the issue that overuse of AMs is a consequence of patient's demand and it is due to their limited understanding of the fact that antibiotics aren't the curative medication for every disease and excessive use has side effects. They also need to know that these drugs have no effect on viral infections. These results were in contrast to a study done in a hospital of Peru that showed patient's pressure to prescribing antimicrobials was considered as a major contributing factor $(72 \%)$ to AM overuse in community. Similarly, a study done in Alexandria university teaching hospital also concluded that antibiotic prescription by physician was influenced by patient's demands (78.5\%) and socioeconomic status $(76.3 \%)$. So, we have concluded that awareness of public through community-targeted media is immensely important. More than half of the participants agreed that AMs in their hospitals are of poor quality. Although we have not explored in detail the definition of "poor" quality. The two main reasons for such substandard quality of medicines include the counterfeit drugs detected in Lahore. Secondly, the use of generic drugs is frequently perceived to be less effective. This affects the physician ability to treat a disease effectively as well as the doctor-patient relationship. The Pakistan Ministry of Health should take some serious action as it is an important issue which directly relates to patient health. The availability of standardized antibiotics should be made possible so that the treatment won't be compromised.

To our best knowledge this is the first multicenter cross-sectional study assessing knowledge, attitude and practice of antimicrobials and antimicrobial resistance of clinicians in Lahore, Pakistan. A uni-center 
study conducted in Services hospital, Pakistan, also concluded that antibiotic overuse has become a major problem as $70 \%$ of physicians were prescribing more than one antibiotic per day and $80 \%$ of the physicians reviewed their decision to prescribe antibiotic by discussing with an experienced colleague that showed that the knowledge of freshly graduated physicians regarding antibiotic prescription, was not up to the mark. Similarly, a study conducted among healthcare workers in Iran showed $88 \%$ agreed on establishment of local guidelines and $94 \%$ believed that education regarding antibiotics can help reduce AMR.

Educational and awareness programs regarding the vigilant use of antimicrobials and AMR should be conducted on regular basis. Mandatory continuing medical education courses should be attended by the healthcare professionals to explain their role in limiting the misuse of antimicrobials and emerging AMR. Moreover, active educational techniques including clinical scenarios that simulate real clinical settings, objective centered clinical examinations, awareness campaigns, and interactive learning workshops should be regularly conducted (Yang, Wu et al. 2016) Future studies from distant, primary and secondary health centers are recommended.

The anonymous nature of questionnaire and voluntary involvement provided reliable answers with no social pressure. Despite the strengths, the study also has some potential limitations. First, a majority of the samples $(74.3 \%)$ was postgraduate trainees in their respective departments. Additionally, a stringent criterion was used to access the knowledge of antimicrobial spectrum which may not be clinically relevant. Another limitation of the study was that the participants from non-teaching hospitals and rural/basic health centers were not interviewed. Moreover, in KAP-survey sometimes the physicians get socially pressurized and give more socially acceptable answers rather than expressing their true opinions. Therefore, to reduce this potential bias, incognito participation was made certain and the case-based questions about AM prescription were composed. Another problem was that consultants working in hospitals did not give due attention to the lengthy questionnaire so it would be apt to say that further studies should be done to access the knowledge, attitudes and practice about antimicrobial use among physicians from community centers. As this study was conducted in six large, public, tertiary-level teaching hospitals, and significant number of qualified prescribing doctors were a part of this, we are positive that the outcomes will be applicable to other public hospitals in Pakistan. Future studies from peripheral health centers are recommended.

\section{Conclusion}

The present KAP-survey has generated information about the knowledge, prescribing attitudes, and practices of medical doctors among all public tertiary care teaching hospitals of Lahore, Pakistan. It highlighted the areas that need improvement to prevent the AMR. The knowledge of clinicians is relatively poor when it comes to drug spectrums and drugs of choice for certain infections. However, the clinicians know about their short comings and they have a positive approach towards professional development.

\section{Declarations}


Acknowledgments: We would like to thank Prof. Ali Ahmad, Department of Microbiology, Infectiology and Immunology, Centre Hospitalier Universitaire (CHU) Sainte Justin/University of Montreal, Montreal, Canada, for his intellectual input and proofreading this article.

Authors Contributions: SoA, ShA, MoA and MAI drafted the first draft and all authors participated in writing subsequent drafts. SoA, MoA, MAI and ZA, AbA, contributed in collecting data and its interpretation. ZA, SoA, MAl and MKA drafted the figures and the Table. AH, QaS, MA, and MI supervised and edited the follow up drafts. All authors approved the final manuscript.

Financial Support: Smile Welfare Organization.

Data Availability: The data used during the current study is available from the corresponding authors on reasonable request.

Competing Interest: Authors declare no competing interest and all the authors have seen and agreed to the submission of this paper.

Ethical Approval: Ethical approval was taken from institutional review board of Shaikh Zayed PostGraduate Medical Institute, Lahore.

Consent to Participate: Written informed consent was taken from participants for data collection and maintaining confidentiality.

Consent to Publish: Not applicable.

\section{References}

Ahmed, H., S. Bhimani, I. Khanum, A. Khan, A. Khetpal, M. A. Abbas, F. Godil, A. Godil and I. U.-H. M. Makhdoom (2020). "Knowledge, attitude and perception survey of doctors regarding antibiotic use and resistance in Karachi, Pakistan." JPMA. The Journal of the Pakistan Medical Association 70(6): 1023.

Anderson, V. R. and C. M. Perry (2008). "Levofloxacin." Drugs 68(4): 535-565.

Cabana, M. D., C. S. Rand, N. R. Powe, A. W. Wu, M. H. Wilson, P.-A. C. Abboud and H. R. Rubin (1999). "Why don't physicians follow clinical practice guidelines?: A framework for improvement." Jama 282(15): 1458-1465.

Cleeland, R. and E. Squires (1984). "Antimicrobial activity of ceftriaxone: a review." The American journal of medicine $77(4 \mathrm{C})$ : 3-11.

Cosgrove, S. E. (2006). "The relationship between antimicrobial resistance and patient outcomes: mortality, length of hospital stay, and health care costs." Clinical Infectious Diseases 42(Supplement_2): S82-S89. 
Costelloe, C., C. Metcalfe, A. Lovering, D. Mant and A. D. Hay (2010). "Effect of antibiotic prescribing in primary care on antimicrobial resistance in individual patients: systematic review and meta-analysis." Bmj 340.

Dellit, T. H., R. C. Owens, J. E. McGowan, D. N. Gerding, R. A. Weinstein, J. P. Burke, W. C. Huskins, D. L. Paterson, N. O. Fishman and C. F. Carpenter (2007). "Infectious Diseases Society of America and the Society for Healthcare Epidemiology of America guidelines for developing an institutional program to enhance antimicrobial stewardship." Clinical infectious diseases 44(2): 159-177.

Faizullah, M., M. I. U. Nisar-ur-Rahman, M. Anwar and M. Sarfraz (2017). "A cross-sectional study on knowledge, attitude and practices of medical doctors towards antibiotic prescribing patterns and resistance in Khyber Pakhtun Khawah, Pakistan." J Appl Pharm Sci 7(12): 38-46.

Freeman, C. D., N. E. Klutman and K. C. Lamp (1997). "Metronidazole." Drugs 54(5): 679-708.

García, C., L. P. Llamocca, K. García, A. Jiménez, F. Samalvides, E. Gotuzzo and J. Jacobs (2011). "Knowledge, attitudes and practice survey about antimicrobial resistance and prescribing among physicians in a hospital setting in Lima, Peru." BMC clinical pharmacology. 11(1): 1-8.

Giblin, T. B., R. L. Sinkowitz-Cochran, P. L. Harris, S. Jacobs, K. Liberatore, M. A. Palfreyman, E. I. Harrison and D. M. Cardo (2004). "Clinicians' perceptions of the problem of antimicrobial resistance in health care facilities." Archives of internal medicine 164(15): 1662-1668.

Guerra, C. M., C. Pereira, A. Neves Neto, D. M. Cardo and L. Correa (2007). "Physicians' perceptions, beliefs, attitudes, and knowledge concerning antimicrobial resistance in a Brazilian teaching hospital." Infect Control Hosp Epidemiol 28(12): 1411-1414.

Lamb, H. M., D. Ormrod, L. J. Scott and D. P. Figgitt (2002). "Ceftriaxone." Drugs 62(7): 1041-1089.

Lartey, P., H. Nellans and S. Tanaka (1994). "New developments in macrolides: structures and antibacterial and prokinetic activities." Advances in Pharmacology_(San Diego,_Calif.). 28: 307-343.

Levy, S. B. (1998). "The challenge of antibiotic resistance." Scientific American 278(3): 46-53.

Marsot, A., A. Boulamery, B. Bruguerolle and N. Simon (2012). "Vancomycin." Clinical pharmacokinetics 51(1): 1-13.

Nathwani, D. (2006). "Antimicrobial prescribing policy and practice in Scotland: recommendations for good antimicrobial practice in acute hospitals." Journal of Antimicrobial Chemotherapy 57(6): 1189-1196.

Okeke, I. N. (2010). Poverty and root causes of resistance in developing countries. Antimicrobial resistance in developing countries, Springer: 27-35. 
Okeke, I. N., R. Laxminarayan, Z. A. Bhutta, A. G. Duse, P. Jenkins, T. F. O'Brien, A. Pablos-Mendez and K. P. Klugman (2005). "Antimicrobial resistance in developing countries. Part l: recent trends and current status." The Lancet infectious diseases 5(8): 481-493.

Organization, W. H. (2012). The evolving threat of antimicrobial resistance: options for action, World Health Organization.

Pulcini, C., E. Cua, F. Lieutier, L. Landraud, P. Dellamonica and P. Roger (2007). "Antibiotic misuse: a prospective clinical audit in a French university hospital." European journal of clinical microbiology \& infectious diseases 26(4): 277-280.

Pulcini, C., F. Williams, N. Molinari, P. Davey and D. Nathwani (2011). "Junior doctors' knowledge and perceptions of antibiotic resistance and prescribing: a survey in France and Scotland." Clinical microbiology and infection 17(1): 80-87.

Rafailidis, P. I., E. N. loannidou and M. E. Falagas (2007). "Ampicillin/sulbactam." Drugs 67(13): 18291849.

Shahid, A., F. Iftikhar, M. K. Arshad, Z. Javed, M. Sufyan, R. S. Ghuman and Z. Tarar (2017). "Knowledge and attitude of physicians about antimicrobial resistance and their prescribing practices in Services hospital, Lahore, Pakistan." J Pak Med Assoc 67(6): 968.

Sosa, A. d. J., D. K. Byarugaba, C. F. Amábile-Cuevas, P.-R. Hsueh, S. Kariuki and I. N. Okeke (2010). Antimicrobial resistance in developing countries, Springer.

Srinivasan, A., X. Song, A. Richards, R. Sinkowitz-Cochran, D. Cardo and C. Rand (2004). "A survey of knowledge, attitudes, and beliefs of house staff physicians from various specialties concerning antimicrobial use and resistance." Archives of internal medicine 164(13): 1451-1456.

Wester, C. W., L. Durairaj, A. T. Evans, D. N. Schwartz, S. Husain and E. Martinez (2002). "Antibiotic resistance: a survey of physician perceptions." Archives of internal medicine 162(19): 2210-2216.

Wilhelm, M. P. and L. Estes (1999). Vancomycin. Mayo Clinic Proceedings, Elsevier.

Woodford, N. and D. M. Livermore (2009). "Infections caused by Gram-positive bacteria: a review of the global challenge." Journal of Infection 59: S4-S16.

Yang, K., D. Wu, F. Tan, S. Shi, X. Guo, Q. Min, X. Zhang and H. Cheng (2016). "Attitudes and perceptions regarding antimicrobial use and resistance among medical students in Central China." Springerplus 5(1): 1779.

Zhanel, G. G., M. Dueck, D. J. Hoban, L. M. Vercaigne, J. M. Embil, A. S. Gin and J. A. Karlowsky (2001). "Review of macrolides and ketolides." Drugs 61(4): 443-498. 
Zhanel, G. G., S. Fontaine, H. Adam, K. Schurek, M. Mayer, A. M. Noreddin, A. S. Gin, E. Rubinstein and D. J. Hoban (2006). "A review of new fluoroquinolones." Treatments in respiratory medicine 5(6): 437-465.

\section{Tables}

\begin{tabular}{|c|c|c|}
\hline \multicolumn{2}{|c|}{ Table 1: Demographic Characteristics of Participants } & \multirow{2}{*}{$\begin{array}{l}\text { Number (percentage) } \\
98(34.5)\end{array}$} \\
\hline Gender & Male & \\
\hline & Female & $186(65.5)$ \\
\hline \multirow[t]{3}{*}{ Years of experience } & $\leq 5$ & 198(69.72) \\
\hline & $5-10$ & $37(13.03)$ \\
\hline & $\geq 10$ & $49(17.25)$ \\
\hline \multirow[t]{2}{*}{ Age } & $\geq 40$ & $47(16.55)$ \\
\hline & $<40$ & $237(83.45)$ \\
\hline \multirow[t]{5}{*}{ Hospital } & $\mathrm{SZH}$ & $58(20.4)$ \\
\hline & JHL & $57(20.1)$ \\
\hline & Mayo & $60(21.1)$ \\
\hline & General & $55(19.4)$ \\
\hline & SIMS & $54(19.0)$ \\
\hline \multirow[t]{5}{*}{ Position } & Post Graduate Trainees & $211(74.3)$ \\
\hline & Medical Officer & $30(10.6)$ \\
\hline & Assistant Professor & 18(6.3) \\
\hline & Associate Professor & $11(3.9)$ \\
\hline & Professor & $14(4.9)$ \\
\hline \multirow[t]{4}{*}{ Department } & Medicine & $82(28.9)$ \\
\hline & Medicine Allied & $93(32.7)$ \\
\hline & Surgery & $47(16.5)$ \\
\hline & Surgery Allied & $62(21.8)$ \\
\hline
\end{tabular}

\section{Figures}


Antimicrobial resistance is a problem worldwide

Cold and flu symptoms will get better after taking antibiotics.

I feel confident about my knowledge and practice in the area of prescribing antibiotics

I believe it is difficult to select the correct antibiotic

It is always better to over-prescribe than under prescribe

I suspect that some antibiotics available in our setup are of poor quality and for that reason do not work

I believe that prescribing antimicrobial does not cause damage when patients do not need them

Need to carry out large scale antibiotic promotion

General knowledge about antimicrobial resistance should be considered when antibiotics are prescribed to an individual patient

Necessary to get more knowledge about antibiotics

Patients demands for antibiotics contribute to overuse

Antimicrobial resistance is not a significant problem in my hospital

Everyone should be allowed to buy antibiotics without prescription.

Need to establish courses on rational use of antibiotics

Efficacy will be better if antibiotics are newer and costly

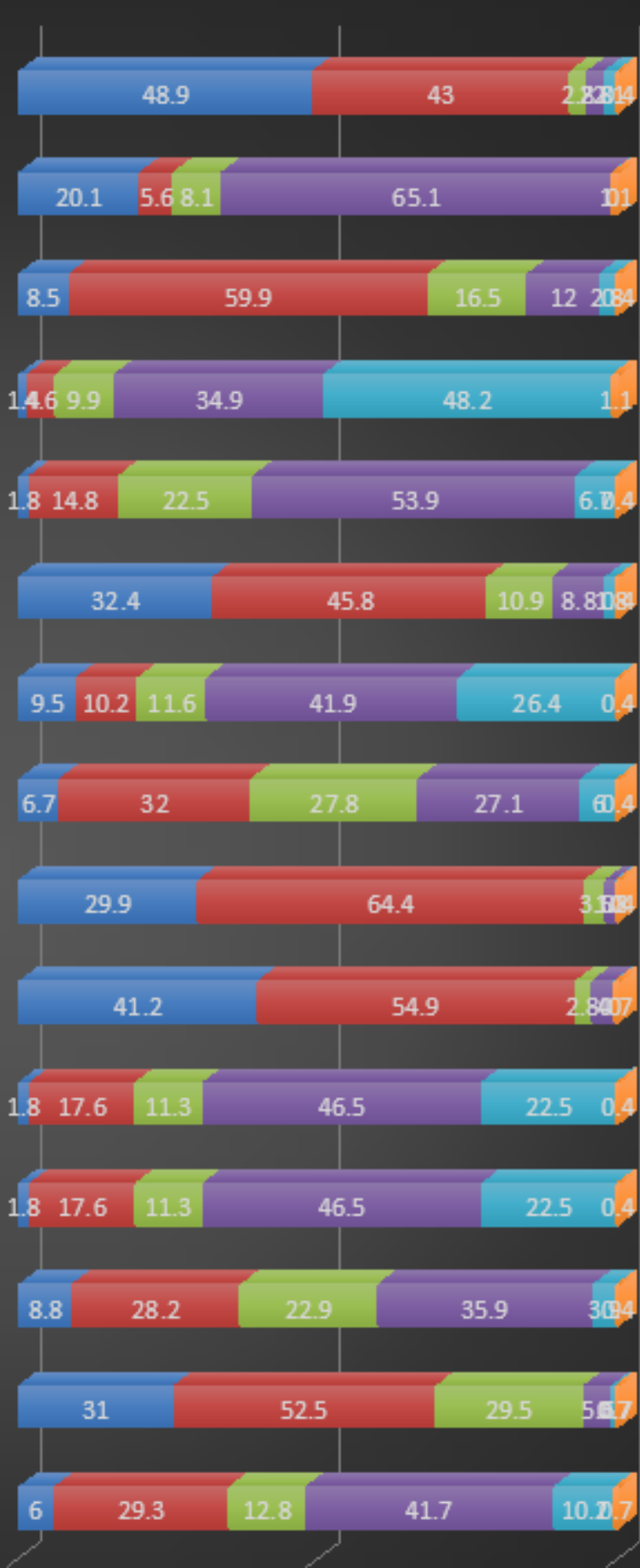

$0 \%$

$50 \%$

$100 \%$

nStrongly Agree $\square$ Agree $\square$ Neutral $\square$ Disagree $\quad$ Strongly Disagree $\square$ Validity

\section{Figure 1}

Awareness of the scope of AMR among 284 participants (data in the graph represents percentages) 


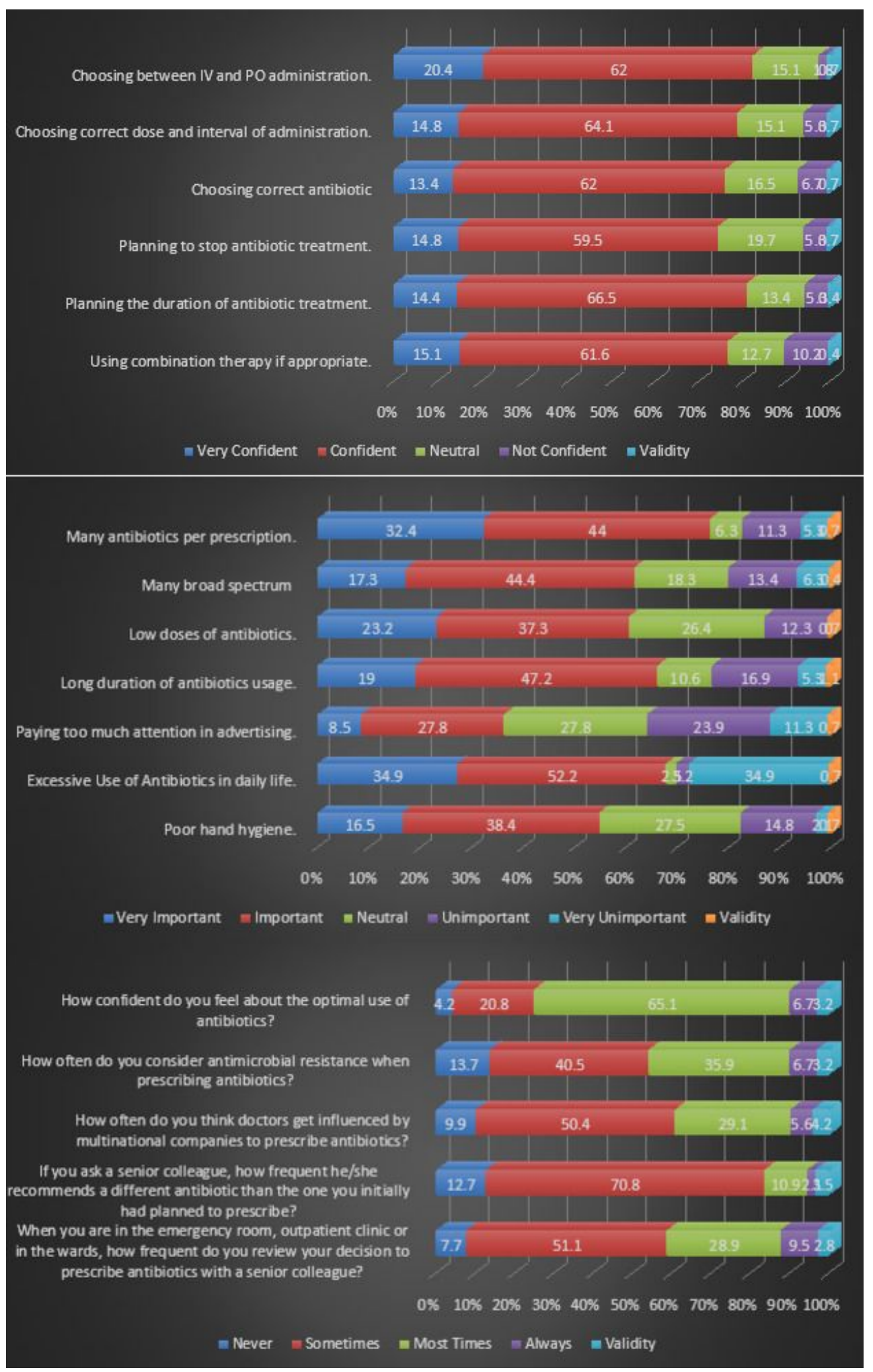

\section{Figure 2}

(A): Factors influencing the clinicians' knowledge leading to AMR. Data represent percentages among 284 participants. (B): Factors influencing the clinicians' practice leading to AMR. Data represent percentages among 284 participants. (C) Factors influencing the clinicians' attitude leading to AMR. Data represent percentages among 284 participants 


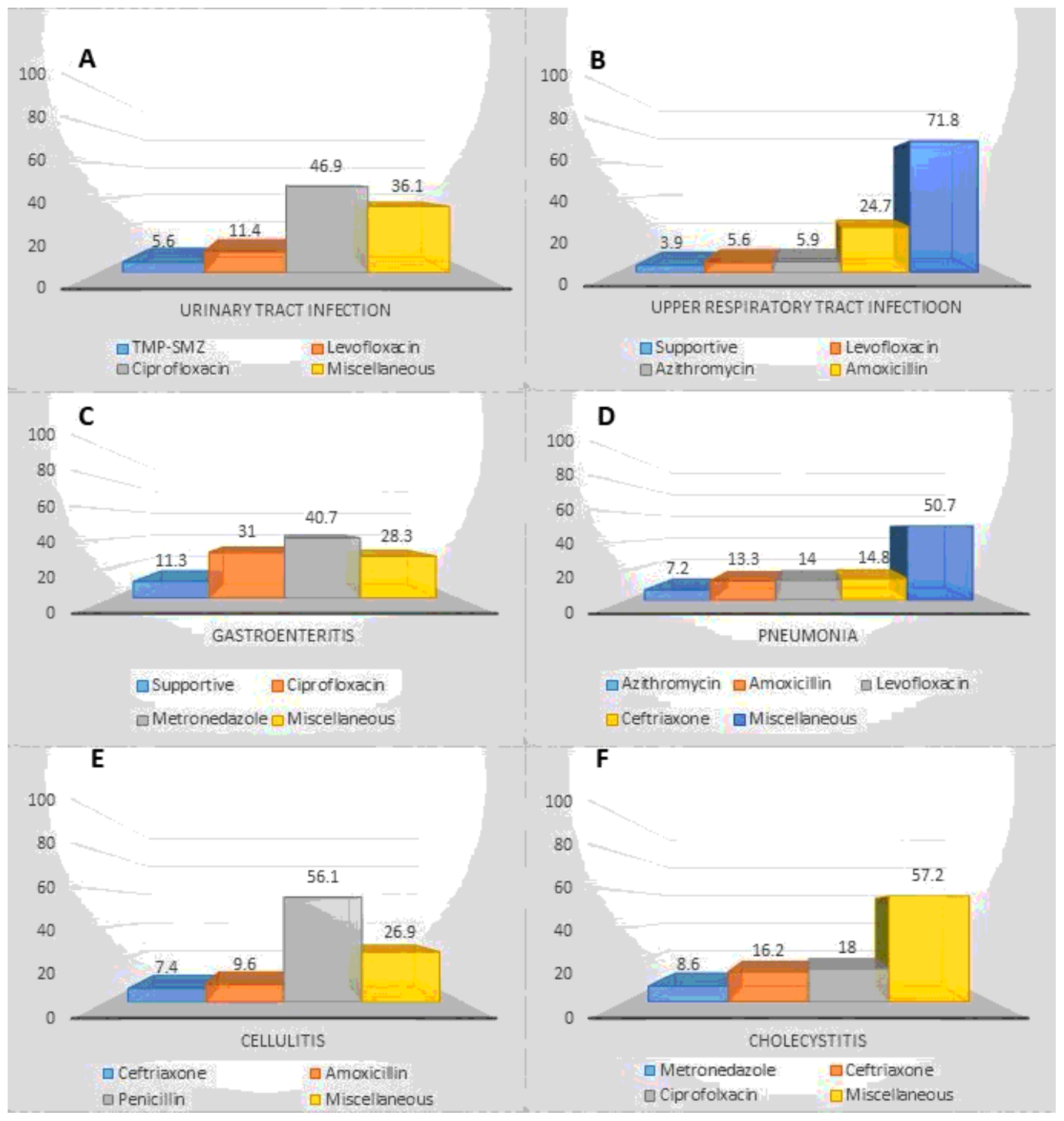

Figure 3

Empirical treatment of various infections. 


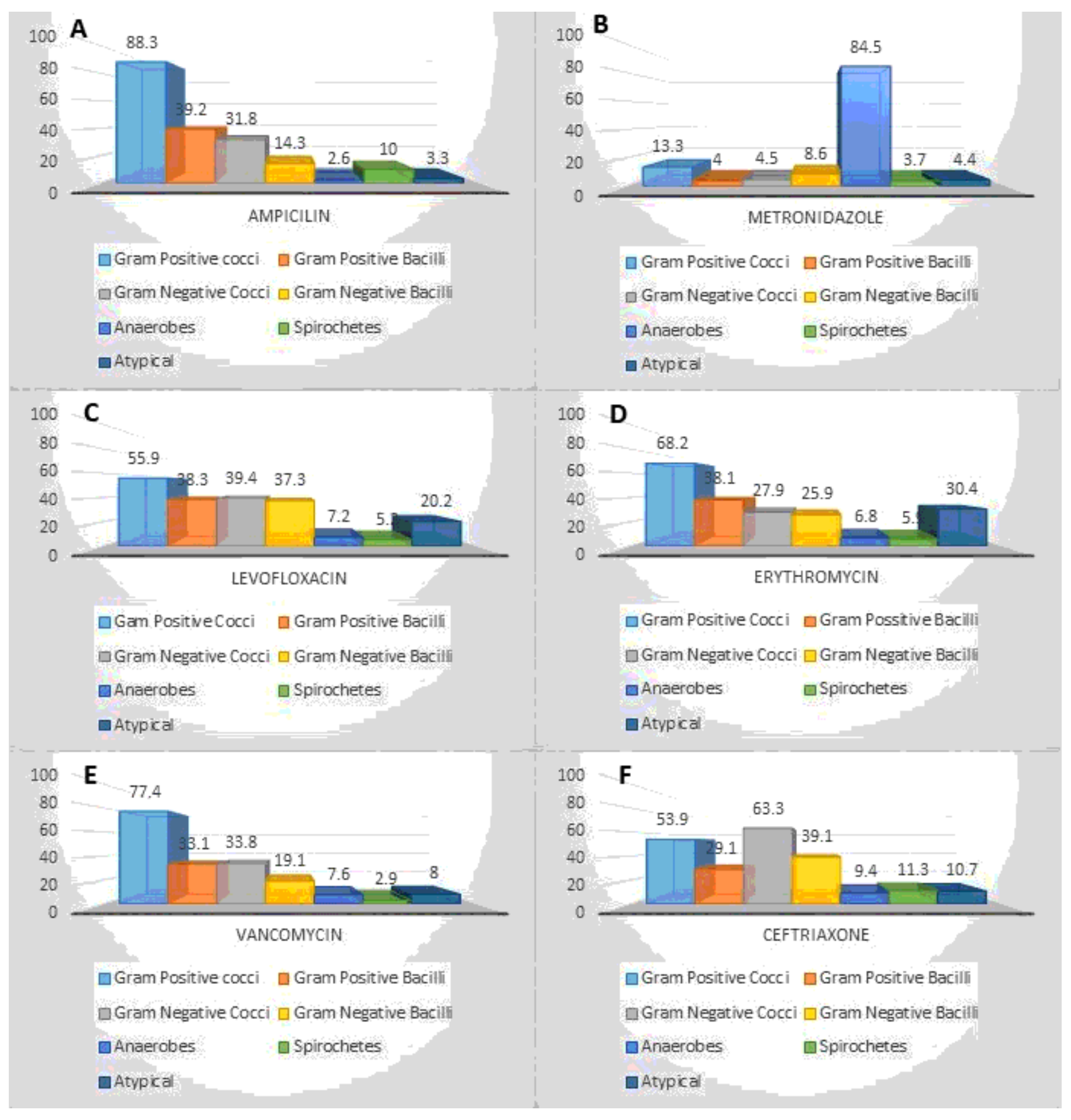

Figure 4

Antimicrobial spectrum of the drugs. 